УДК 621.311

\title{
АНАЛИЗ ПОТЕРЬ ЭЛЕКТРОЭНЕРГИИ В СИСТЕМЕ ЭЛЕКТРОСНАБЖЕНИЯ ОБОГАТИТЕЛЬНОЙ ФАБРИКИ
}

\author{
Клюев Роман Владимирович1, \\ kluev-roman@ramler.ru
}

\section{Голик Владимир Иванович²,} v.i.golik@mail.ru

\author{
Босиков Игорь Иванович², \\ igor.boss.777@mal.ru
}

\author{
Гаврина Оксана Александровна², \\ Gavrina-Oksana@yandex.ru \\ 1 Московский политехнический университет, \\ Россия, 107023, г. Москва, ул. Б. Семеновская, 38 \\ 2 Северо-Кавказский горно-металлургический институт (государственный технологический университет), \\ Россия, 362021, г. Владикавказ, ул. Николаева, 44.
}

\begin{abstract}
Актуальность работы обусловлена необходимостью комплексного исследования системы электроснабжения обогатительных фабрик. Для этого следует разработать эфффективную методику расчета потерь электроэнергии по отдельным технологическим механизмам и фабрике в целом, что даст возможность существенно сократить энергетическую составляющую затрат в ходе выпуска продукции.

Цель: разработать методику определения потерь электроэнергии по отдельным технологическим механизмам в различных режимах работы, которая может быть использована на аналогичных предприятиях.

объекты: горно-обогатительные фабрики, которые характеризуются сложностью определения энергетических характеристик технологического оборудования.

Методы: совокупность детерминированных и вероятностно-статистических методов определения потерь электроэнергии. Детерминированные методы включают расчет максимальных потерь, средних нагрузок, графического интегрирования, среднеквадратичного тока. В свою очередь, вероятностно-статистические основаны на исследовании статистических характеристик и применении статистических выборок схем сети.

Результаты. На основании экспериментальных исследований разработана методика определения потерь электроэнергии в элементах системы электроснабжения обогатительной фабрики в номинальном и в эксплуатационном режиме. Установлено, что отношение коэффициентов потерь в номинальном и эксплуатационном режимах работы, определяющее степень использования пропускной способности кабельной сети 6 кВ, находится в пределах 1,02-2,57. В среднем кабельная сеть 6 кВ недогружена на 25-30 \%. Фактические потери электроэнергии в кабельной сети 6 кВ оказались меньше номинальных и составляют менее $1 \%$ от общей потребляемой электроэнергии, следовательно, проведение каких-либо мероприятий для уменьщения потерь электроэнергии нецелесообразно. Средневзвешенный коэфффициент мощности по обогатительной фрабрике равен 0,82, поэтому может оказаться целесообразным проведение мероприятий по компенсации реактивной мощности. Расчет параметров компенсирующих устройств и выбор места их установки предусмотрены планом работ на будущий период.
\end{abstract}

\section{Ключевые слова:}

Обогатительная фабрика, потери электроэнергии, кабельная сеть,

система электроснабжения, метод графического интегрирования, мощность оборудования.

\section{Введение}

При разработке месторождений полезных ископаемых показатели эффективности производства во многом зависят от потребления электроэнергии, особенно на стадии обогащения руд с использованием мощного энергонасыщенного оборудования. Резервом снижения потребления электроэнергии является минимизация ее потерь в энергозатратных процессах, например, механохимическом извлечении металлов из руд [1], а также повышении эффективности ее использования [2-9].

Рассматриваемая в работа обогатительная фабрика включает следующие технологические переделы:

1) измельчение баритовых и меднопиритовых руд;

2) дробление руды;
3) транспортировка руды, реагентное отделение;

4) флотация;

5) фильтрация и сушка;

6) известковое отделение;

7) компрессорная;

8) хвостовое хозяйство.

В ходе выпуска продукции основным технологическим оборудованием являются шаровые мельницы, дробилки и насосы.

Потери электроэнергии должны систематически контролироваться и поддерживаться в экономически целесообразных границах. Контроль за величиной потерь электроэнергии ведется на каждом участке сети поэлементно. Выявленные участки сети с повышенными потерями против рациональных значений потерь электроэнергии должны быть реконструированы. 
Наиболее часто применяется метод графического интегрирования, при котором, имея характер изменения протекающего по линии тока во времени в виде графика в координатных осях $I, t$, можно определить потери электроэнергии графическим путем; метод среднеквадратичного тока, в котором действительный переменный по величине ток заменяется неизменным

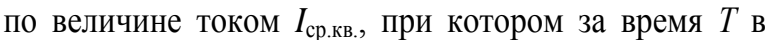
линии выделяются такие же потери энергии. Известна также модификация этих методов $[10,11]$.

\section{Выбор методики определения потерь электроэнергии}

Для расчета потерь в радиальных сетях 6 кВ обогатительной фабрики ГОК используем совокупность некоторых методов, которые используются различными учеными [12-20]. Величина времени потерь электроэнергии определится по годовому графику нагрузки по продолжительности. Этот график получается из действительного годового графика нагрузки, имеющего самую причудливую форму, перестроением последнего в порядке убывания его координат.

Потери электроэнергии определяются по формуле:

$$
\Delta W=3 R \int_{0}^{T} I_{t}^{2} d t=3 R \sum_{i=1}^{n} I_{t}^{2} \Delta t=3 R \frac{t}{n} \sum_{i=1}^{n} I_{t}^{2} \cdot 10^{-3},
$$

где $n$ - число равных интервалов времени $\Delta t ; R$ - эквивалентное активное сопротивление линии; $t$ - текущее время; $I_{t}=f(t)-$ график нагрузки.

При использовании неизменной максимальной нагрузки такое же количество нагрузочных потерь выделится за меньшее время. Это время называется временем максимальных потерь $\left(\tau_{\max }\right)$.

С учетом использования ( $\left.\tau_{\max }\right)$ формулу $(1)$ можно записать следующим образом:

$$
\Delta W=3 R \int_{0}^{T} I_{t}^{2} d t=3 R I_{\max }^{2} \tau=\Delta P_{\max } \tau,
$$

где $\Delta P_{\max }$ - величина максимальных потерь активной мощности.

Откуда:

$$
\tau_{\max }=\frac{\int_{0}^{T} I_{t}^{2} d t}{I_{\max }^{2}} .
$$

Вторая зависимость определяет связь между временем потерь и параметрами годового графика активной мощности:

$$
\tau_{\max }=2 T_{\max }-8760+\frac{8760-T_{\max }}{1+\frac{T_{\max }}{8760}-\frac{2 P_{\min }}{P_{\max }}}\left(1-\frac{P_{\min }}{P_{\max }}\right) .
$$

Однако для определения $\tau_{\max }$ по этой формуле требуется дополнительная информация, определение которой связано с некоторыми трудностями.

Для оценки потерь энергии в радиальных сетях, питающих чисто промышленную нагрузку, достаточную точность дают кривые, построенные А.А. Глазуновым. Здесь $\tau_{\max }$ определяются в зависимости от времени использования максимальной нагрузки $T_{\max }$ и коэффициента мощности $\cos \varphi$.

Значения годового числа использования максимальной нагрузки $T_{\max }$ целесообразно принимать не среднестатическими, а на основании действительных данных. Зная расход электроэнергии оборудования за текущий период (например, за год), номинальную мощность и учитывая, что в рабочем режиме электрооборудование загружено не на номинальную мощность, можно принять, что:

$$
P_{\max }=P_{\text {ном }}
$$

Тогда

$$
T_{\text {max }}=\frac{W_{\text {раб }}}{P_{\text {ном }}} .
$$

Действительную загрузку за отчетный период можно определить, зная расход электроэнергии за период и среднее время работы оборудования за этот же период

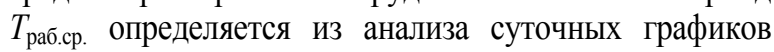
нагрузки, поэтому в качестве отчетного периода принимаются одни сутки. Формула принимает вид:

$$
P_{\text {раб }}=\frac{W_{\text {раб }}}{365 T_{\text {раб. ср }}} .
$$

При снижении мощности асинхронного двигателя уменьшается его коэффициент мощности $(\cos \varphi)$ :

$$
\cos \varphi=\frac{P}{S}=\frac{1}{\sqrt{1+\left(\frac{Q}{P}\right)^{2}}},
$$

где $P$ и $Q$ - соответственно, активная и реактивная мощности, потребляемые двигателем.

При номинальной загрузке потребляемая реактивная мощность:

$$
Q_{\mathrm{H}}=P_{\mathrm{H}} \operatorname{tg} \varphi_{\mathrm{H}} .
$$

Для двигателей с $\cos \varphi=0,77 \ldots 0,79$ реактивная мощность холостого хода составляет около $70 \%$ реактивной мощности:

$$
Q_{\mathrm{xx}} \approx 0,7 Q_{\mathrm{H}}
$$

Увеличение потребления реактивной мощности при полной загрузке двигателя по сравнению с потреблением при холостом ходе:

$$
\Delta Q_{\mathrm{H}}=Q_{\mathrm{H}}-Q_{\mathrm{xx}}
$$

При нагрузке менее номинальной прирост потребления реактивной мощности по сравнению с холостым ходом пропорционален квадрату коэффициента загрузки двигателя:

$$
\Delta Q=k_{3}^{2} \Delta Q_{\mathrm{H}}
$$

где $k_{3}^{2}=\frac{P}{P_{\text {н }}}-$ коэффициент загрузки двигателя.

Таким образом, реактивная мощность, потребляемая двигателем при произвольной загрузке, составляет:

$$
Q=Q_{\mathrm{xx}}+k_{3}^{2} \Delta Q_{\mathrm{H}}
$$


С учетом (6)-(10) выражение (5) запишется следующим образом:

$$
\cos \varphi=\frac{1}{\sqrt{1+\left(\frac{Q_{x x}+k_{3}^{2} \Delta Q_{\mathrm{H}}}{P}\right)^{2}}}=
$$$$
=\frac{1}{\sqrt{1+\left(\frac{P_{\mathrm{H}} \operatorname{tg} \varphi_{\mathrm{H}} \cdot\left[0,7+0,3\left(\frac{P}{P_{\mathrm{H}}}\right)^{2}\right]}{P}\right)^{2}}} .
$$

Для двигателей с номинальным коэффициентом мощности: $\quad \cos \varphi_{\mathrm{H}}=0,8 \ldots 0,9 ; \quad Q_{\mathrm{xx}} \approx 0,6 Q_{\mathrm{H}} . \quad$ А для: $\cos \varphi_{\mathrm{H}}=0,91 \ldots 0,93 ; Q_{\mathrm{xx}} \approx 0,5 Q_{\mathrm{H}}$.

Поэтому формула (5) для них запишется соответственно:

$$
\begin{aligned}
& \cos \varphi=\frac{1}{\sqrt{1+\left(\frac{P_{\mathrm{H}} \operatorname{tg} \varphi_{\mathrm{H}}\left[0,6+0,4\left(\frac{P}{P_{\mathrm{H}}}\right)^{2}\right]}{P}\right)^{2}}} \\
& \cos \varphi=\frac{1}{\sqrt{1+\left(\frac{P_{\mathrm{H}} \operatorname{tg} \varphi_{\mathrm{H}}\left[0,5+0,5\left(\frac{P}{P_{\mathrm{H}}}\right)^{2}\right]}{P}\right)^{2}}} .
\end{aligned}
$$

Величина потерь активной энергии может быть подсчитана по известным значениям среднеквадратичного тока:

$$
\Delta W=3 R \int_{0}^{t} I_{t}^{2} d t=3 R I_{\text {ср. кв }}^{2} T,
$$

где $I_{\text {ср. кв }}-$ значение среднеквадратичного тока.

Откуда:

$$
I_{\text {ср. кв }}=\sqrt{\frac{\int_{0}^{T} I_{t}^{2} d t}{T} .}
$$

Среднеквадратичный ток - это такой условный ток, неизменный по величине, при протекании которого по линии в течение времени $T$ создаются такие потери, какие линия имеет при работе по действительному графику нагрузки за то же время.

Выразив $\int_{0}^{T} I_{t}^{2} d t$ из выражения (3) и подставив его в (12), можно получить следующее выражение для среднеквадратичного тока:

$$
I_{\text {ср. Кв }}=\sqrt{\frac{I_{\max }^{2} \tau}{T}}=I_{\max } \sqrt{\frac{\tau}{T}} .
$$

C учетом выражения (13) величина потерь активной энергии будет равна:

$$
\Delta W=3 I_{\text {cp. кв }}^{2} R T=3 I_{\max }^{2} R \tau=\Delta P \tau,
$$

где $\Delta P$ - потери мощности в кабельной сети.

Преобразуя выражение (14) к виду, удобному для расчетов, получим:

$$
\Delta W=3 R \sum_{i=1}^{n} I_{\mathrm{cp.K \textrm {k }}_{i}}^{2} T_{i}=\frac{R}{\left(U_{\mathrm{H}} \cos \varphi\right)^{2}} \sum_{i=1}^{n} \frac{W_{i}}{T_{i}},
$$

где $R$ - активное сопротивление кабеля, Ом; $U_{\text {н }}-$ номинальное напряжение сети, $\mathrm{\kappa}$; $\cos \varphi$ - действительный коэффициент мощности; $W_{i}$ - расход электроэнергии за расчетный период, кВт.ч; $T_{i}-$ расчетный период, ч.

При расчете потерь электрической энергии в трансформаторах, важнейшими факторами являются: фактическая загрузка трансформаторов, число параллельно работающих трансформаторов, не отключаемых при снижении нагрузки, и годовые потери холостого хода.

Для трехфазных трехобмоточных трансформаторов потери электроэнергии рассчитываются по предложенным А.А. Федоровым формулам, где предполагается, что $k_{3}$ при включении $n$ трансформаторов увеличивается соответственно в $n$ раз, т. е. $k_{3}=$ const:

$$
\begin{gathered}
\Delta W=n \Delta P_{\mathrm{xx}} T+ \\
+\frac{1}{n}\left[k_{3 \mathrm{BH}}^{2} \Delta P_{\mathrm{rзBH}}+k_{3 \mathrm{CH}}^{2} \Delta P_{\mathrm{кз} \mathrm{CH}}+k_{3 \mathrm{HH}}^{2} \Delta P_{\mathrm{rзHH}}\right] \tau .
\end{gathered}
$$

При $k_{3}=$ var формула имеет вид:

$$
\begin{gathered}
\Delta W=n \Delta P_{\mathrm{xx}} T+ \\
+\left[\left(\frac{k_{3 \mathrm{BH}}^{2}}{n}\right) \Delta P_{\mathrm{kзBH}}+\left(\frac{k_{3 \mathrm{CH}}^{2}}{n}\right) \Delta P_{\mathrm{k3} \mathrm{CH}}+\left(\frac{k_{3 \mathrm{HH}}^{2}}{n}\right) \Delta P_{\mathrm{k} 3 \mathrm{HH}}\right] \tau,
\end{gathered}
$$

где $k_{3}$ - коэффициент загрузки соответствующей обмотки; $n$ - число параллельно работающих трансформаторов; $\Delta P_{\mathrm{xx}}-$ потери мощности для режимов холостого хода; $\Delta P_{\text {кз }}$ - потери мощности для режима короткого замыкания соответствующей обмотки.

Потери мощности в металле обмоток определяются следующими выражениями:

$$
\begin{aligned}
\Delta P_{\mathrm{r} 3 \mathrm{BH}} & =\frac{\Delta P_{\mathrm{r} 3 \mathrm{BH}-\mathrm{CH}}+\Delta P_{\mathrm{\kappa} 3 \mathrm{BH}-\mathrm{HH}}-\Delta P_{\mathrm{r} 3 \mathrm{CH}-\mathrm{HH}}}{2} . \\
\Delta P_{\mathrm{r} 3 \mathrm{CH}} & =\frac{\Delta P_{\mathrm{r} 3 \mathrm{BH}-\mathrm{CH}}+\Delta P_{\mathrm{r} 3 \mathrm{CH}-\mathrm{HH}}-\Delta P_{\mathrm{k} 3 \mathrm{BH}-\mathrm{HH}}}{2} . \\
\Delta P_{\mathrm{r} 3 \mathrm{HH}} & =\frac{\Delta P_{\mathrm{r} 3 \mathrm{BH}-\mathrm{HH}}+\Delta P_{\mathrm{r} 3 \mathrm{CH}-\mathrm{HH}}-\Delta P_{\mathrm{r} 3 \mathrm{BH}-\mathrm{CH}}}{2} .
\end{aligned}
$$

Величина потерь для номинального и расчетного режима работы элементов электрической сети оценивается по значению коэффициентов потерь активной энергии. 
Значение коэффициента потерь в номинальном режиме работы элемента или участка электрической сети определяется по формуле:

$$
K_{n W_{\mathrm{H}}}=\frac{\Delta W_{\mathrm{H}}}{W_{\mathrm{H}}}=\frac{\Delta P_{\mathrm{H}}}{\sum_{i=1}^{n} P_{\mathrm{H} i}} \cdot \frac{\tau}{T_{\max }},
$$

где $\Delta P_{\mathrm{H}}$ - суммарные потери активной мощности в номинальном режиме работы, кВт; $\sum_{i=1}^{n} P_{\text {н }}$ - суммарная номинальная активная мощность группы элементов или участка сети, кВт; $\tau$ - продолжительность потерь активной мощности в течение года, ч; $T_{\max }$ продолжительность использования максимума активной нагрузки, ч.

Значение коэффициента потерь в рабочем режиме элемента и участка электросети определяется по формуле:

$$
K_{n W_{\mathrm{p}}}=\frac{\Delta W_{\mathrm{p}}}{W_{\mathrm{p}}}=\frac{\Delta P_{\mathrm{p}}}{\sum_{i=1}^{n} P_{\mathrm{p} i}} \cdot \frac{\tau}{T_{\max }},
$$

где $\Delta P_{\mathrm{p}}$ - суммарные потери активной мощности в рабочем режиме работы, кВт; $\sum_{i=1}^{n} P_{\mathrm{p} i}$ - суммарная рабочая активная мощность группы элементов или участка сети, кВт:

$$
\sum_{i=1}^{n} P_{\mathrm{p} i}=3 \sum_{i=1}^{n} I_{\mathrm{p} i}^{2} R_{i} .
$$

По соотношению коэффициентов расчетных и номинальных потерь судят об использовании возможностей элементов системы электроснабжения:

$$
K_{n}^{*}=\frac{K_{n W_{\mathrm{p}}}}{K_{n W_{\mathrm{H}}}}=\frac{\Delta P_{\mathrm{p}} \sum_{i=1}^{n} P_{\mathrm{н} i}}{\Delta P_{\mathrm{H}} \sum_{i=1}^{n} P_{\mathrm{p} i}} .
$$

C помощью коэффициента расчетных потерь группы элементов или участка электрической сети определяются величины потерь активной энергии за рассматриваемый интервал времени по величине потребляемой активной мощности или энергии:

$$
\Delta W=\frac{\Delta P_{\mathrm{p}} \tau}{\sum_{i=1}^{n} P_{\mathrm{p} i} T_{\max }} W_{\text {потр }}=K_{n W_{\mathrm{p}}} W_{\text {потр }} .
$$

\section{Расчет потерь электроэнергии}

Потери электрической энергии в кабельных сетях складываются из потерь в каждом отдельном кабеле, расчет которых проводится по вышеприведенной методике.

В качестве примера рассмотрим расчет потерь электроэнергии в кабеле типа ААБГВ $(3 \times 70)$ длиной $l=400$ м, питающем дробилку № 1 от ГРУ -6 кВ.

1. Активное сопротивление кабеля:

$$
R=r_{0} \cdot l,
$$

где $r_{0}$ - погонное сопротивление, Ом/км; $l$ - длина кабеля, км.

Погонное сопротивление всех кабелей с алюминиевыми жилами сечением $70 \mathrm{mм}^{2}: r_{0}=0,447$ Ом/км при $x_{0}=0,08 \mathrm{OM} / \mathrm{Kм}$

$$
R=0,447 \cdot 0,4=0,179 \text { Ом. }
$$

2. На основании данных по расходу электроэнергии за 2019 г. определим расход электроэнергии в рабочем режиме за 2019 г.: $W_{\text {раб }}=154440$ кВт.ч.

3. По суточным графикам нагрузок определим среднее время работы оборудования за сутки: $T_{\text {раб.ср }}=3$ ч.

4. Определим годовое число часов использования максимальной активной нагрузки:

$$
T_{\text {max }}=\frac{W_{\text {раб }}}{P_{\text {ном }}},
$$

где $P_{\text {ном }}-$ номинальная мощность двигателя дробилки, кВТ.

$$
T_{\max }=\frac{154440}{250}=620 \text { ч. }
$$

5. По годовому числу часов использования максимальной нагрузки $T_{\max }=620$ ч и коэффициенту мощности двигателя $\cos \varphi_{\mathrm{H}}=0,78$ определяем значение времени максимальных потерь: $\tau=f\left(T_{\max }\right.$, $\cos \varphi_{\text {н }}$, равное $\tau=500$ ч.

6. По формуле (2) определим потери мощности в кабеле в номинальном режиме работы дробилки:

$$
\Delta P_{\text {ном }}=3 R \cdot I_{\text {ном }}^{2} \cdot 10^{-3}=0,585 \text { кВТ. }
$$

Соответственно потери электроэнергии в кабеле в номинальном режиме работы дробилки:

$$
\Delta W_{\text {ном }}=\Delta P_{\text {ном }} \cdot \tau=0,585 \cdot 500=295,5 \text { кВТ } \cdot \text { ч. }
$$

7. Рассчитаем среднюю рабочую мощность электродвигателя за сутки:

$$
P_{\text {раб }}=\frac{W}{T}=\frac{154440}{365 \cdot 3}=141 \kappa \text { кВТ. }
$$

8. Потери электроэнергии в рабочем режиме определим из соотношения (15). Потери электроэнергии определим по данным 2019 г. Расчетный период составляет $T=365 \cdot T_{\text {раб.ср. }}$

Действительный коэффициент мощности двигателя определим по выражению (11), так как $\cos \varphi_{\mathrm{H}}=0,78$ :

$$
\begin{gathered}
\cos \varphi=\frac{1}{\sqrt{1+\left(\frac{250 \cdot 0,8023 \cdot\left[0,7+0,3 \cdot\left(\frac{141}{250}\right)^{2}\right]}{141}\right)^{2}}}=0,66 . \\
\Delta W_{\text {раб }}=\frac{0,179}{(6 \cdot 0,66)^{2}} \cdot \frac{154440^{2}}{365 \cdot 3} \cdot 10^{-3}=248,6 \text { кВТ } \cdot \text { ч. }
\end{gathered}
$$

9. Потери мощности в данном кабеле в рабочем режиме будут равны:

$$
\Delta P_{\text {раб }}=\frac{\Delta W_{\text {раб }}}{\tau}=\frac{248,6}{500}=0,497 \kappa \text { кВ . }
$$


10. Рассчитаем действительный коэффициент использования оборудования:

$$
k_{\text {и }}=\frac{P_{\text {раб }}}{P_{\text {ном }}}=\frac{141}{250}=0,56 .
$$

11. Определим рабочий ток двигателя:

$$
I_{\text {раб }}=\frac{P_{\text {раб }}}{\sqrt{3} U_{\mathrm{H}} \cos \varphi_{\mathrm{H}} \eta},
$$

где $\eta$ - КПД двигателя.

$$
I_{\text {раб }}=\frac{141}{\sqrt{3} \cdot 6 \cdot 0,66 \cdot 0,95}=22 \mathrm{~A} .
$$

12. Определим коэффициент потерь мощности в номинальном режиме:

$$
K_{\text {п.н }}=\frac{\Delta P_{\mathrm{H}}}{P_{\mathrm{H}}}=\frac{0,585}{250}=0,234 \cdot 10^{-2} .
$$

13. Коэффициент потерь мощности в рабочем режиме:

$$
K_{\text {п.р }}=\frac{\Delta P_{\text {раб }}}{P_{\text {раб }}}=\frac{0,497}{141}=0,352 \cdot 10^{-2} .
$$

14. Относительный коэффициент потерь активной мощности:

$$
K^{*}=\frac{K_{\text {п.р }}}{K_{\text {п.н }}}=\frac{0,352 \cdot 10^{-2}}{0,234 \cdot 10^{-2}}=1,5 .
$$

Аналогичным образом определяются потери мощности и электроэнергии в кабельных сетях 6 кВ по всем высоковольтным приемникам обогатительной фабрики.

Значения потерь мощности для отдельных механизмов обогатительной фабрики приведены на рисунке.

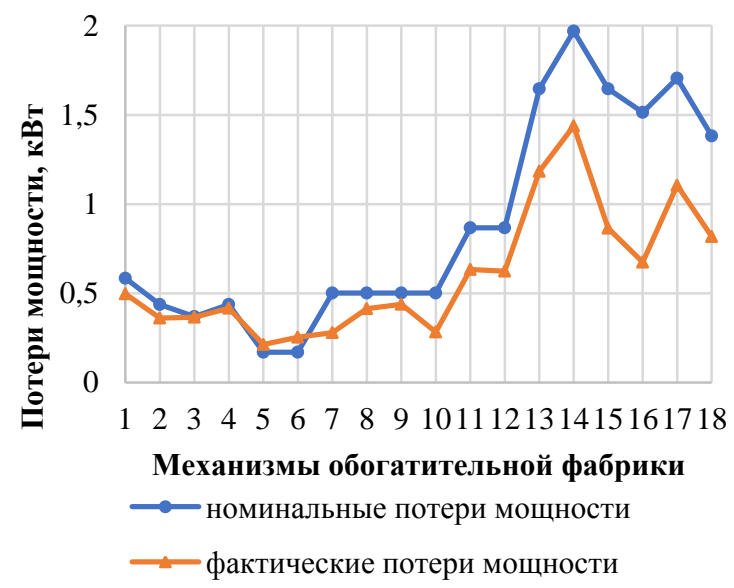

Рисунок. Значения потерь мощности для отдельных механизмов обогатительной фабрики: 1-4 дробилки № 1-4; 5, 6 - шаровые мельницы № 2, 3; 7-12 - насосы № 1-6; 13-18 - шаровые мельниubl № 4-10

Figure. Power loss values for individual mechanisms of the processing plant: 1-4-crushers no. 1-4; 5, 6-ball mills no. 2, 3; 7-12 - pumps no. 1-6; 13-18-ball mills no. $4-10$
Как видно из рисунка, фактические потери мощности почти у всех потребителей оказываются меньше номинальных. Исключение составляют только двигатели привода шаровых мельниц № 2, 3. Так как планом развития предусмотрен переход с переработки баритосодержащих руд на золотосодержащие руды, то мельницы № 2, 3 демонтируются. Поэтому проведение каких-либо мероприятий для уменьшения потерь электроэнергии в этих мельницах нецелесообразно.

Определим потери мощности и энергии в кабелях 6 кВ от трансформаторов ГПП до ГРУ напряжением 6 кB.

На ГПП рабочим является один трансформатор; второй служит резервом. Кроме того, в работе может находиться либо один ввод ГРУ, и тогда оставшаяся часть потребителей ГРУ запитывается через межсекционный выключатель; либо включены оба ввода, и секции ГРУ работают раздельно. При работающем одном вводе ГРУ энергия может передаваться по двум или трем ниткам кабелей (в зависимости от работающего трансформатора).

Потери определим для трех случаев: а) работает один ввод и две нитки кабеля; б) работает один ввод и три нитки кабеля; в) работают два ввода и обе нитки кабелей.

Активное сопротивление кабелей определяется по выражению (20). Погонное сопротивление кабелей с алюминиевыми жилами сечением $185 \mathrm{~mm}^{2}$ при $r_{0}=0,167 \mathrm{Oм} / \mathrm{\kappa м} ; x_{0}=0,073 \mathrm{OM} / \mathrm{\kappa м}$.

a) $R_{1}=0,167 \cdot 0,8 \cdot \frac{1}{2}=0,067 \mathrm{OM}$;

б) $R_{2}=0,167 \cdot 0,8 \cdot \frac{1}{3}=0,045 \mathrm{OM}$;

в) $R_{3}=0,167 \cdot 0,8 \cdot \frac{1}{5}=0,027$ Ом.

Определим суммарный расход электроэнергии всех потребителей 6 кВ за 2019 г.:

$$
W_{\Sigma}=\sum_{i=1}^{n} W_{i}=39388080 \text { кВт } \cdot \text { ч. }
$$

Средневзвешенный коэффициент мощности:

$$
\cos \varphi_{\text {ср. в3 }}=\frac{\sum_{i=1}^{n} P_{\mathrm{p} i} \cdot \cos \varphi_{i}}{\sum_{i=1}^{n} P_{\mathrm{p} i}}=\frac{6758,3}{8237}=0,82 .
$$

Определим средневзвешенный коэффициент использования всего оборудования, подключенного к ГРУ напряжением 6 кВ:

$$
k_{\text {и ср. вз }}=\frac{\sum_{i=1}^{n} k_{\text {иi }} P_{\mathrm{p} i}}{\sum_{i=1}^{n} P_{\mathrm{p} i}}=\frac{5562,34}{8237}=0,68 .
$$

Суммарный ток, протекающий от ГПП к ГРУ в рабочем режиме с учетом коэффициента использования определится: 


$$
I_{\Sigma \text { раб }}=k_{\text {и ср.вз }} \sum_{i=1}^{n} I_{\text {iр }}=0,68 \cdot 1054,2=716,9 \text { A. }
$$

Потери мощности определим по выражению (2):

а) $\Delta P_{1}=3 \cdot 716,9^{2} \cdot 0,067=103,3 \mathrm{\kappa BT}$;

б) $\Delta P_{2}=3 \cdot 716,9^{2} \cdot 0,045=69,4 \mathrm{\kappa BT}$;

в) $\Delta P_{3}=3 \cdot 716,9^{2} \cdot 0,027=41,6$ кВТ.

Годовое число часов использования максимальной активной нагрузки всех приемников определим по выражению (4): $T_{\max }=\frac{W_{\Sigma}}{P_{\max }}$. Максимальную мощность принимаем равной $P_{\max }=6,3$ МВт на основе анализа данных тридцатиминутной максимальной нагрузки за 2019 г. Тогда:

$$
T_{\max }=\frac{39388080}{6300}=6200 \text { ч. }
$$

По годовому числу часов использования активной нагрузки и средневзвешенному коэффициенту мощности оборудования определим значение времени максимальных потерь $\tau=f\left(T_{\max } ; \quad \cos \varphi_{\text {ср.вз. }}\right)$, равное $\tau=5000$ ч. Определим потери электроэнергии в кабелях ввода по (14):

a) $\Delta W_{\text {раб.1 }}=103,3 \cdot 5000=516500 \mathrm{\kappa BT} \cdot$ ч;

б) $\Delta W_{\text {ра..2 }}=69,4 \cdot 5000=347000 \kappa \mathrm{BT} \cdot \mathrm{ч}$;

в) $\Delta W_{\text {раб.3 }}=41,6 \cdot 5000=208000 \mathrm{\kappa BT} \cdot ч$.

Для снижения потерь электроэнергии в кабельных линиях от трансформаторов ГПП до ГРУ напряжением 6 кВ целесообразно, чтобы в работе находились оба ввода и кабельные линии работали параллельно.

При работе одного ввода и трех ниток кабелей потеря энергии составит

$$
\begin{gathered}
\Delta W_{1}=\Delta W_{\text {раб. } 2}-\Delta W_{\text {раб.3 }}= \\
=347000-208000=139000 \kappa \mathrm{BT} \cdot \mathrm{ч},
\end{gathered}
$$

или $67 \%$ от оптимального режима

При работе одного ввода и двух ниток кабелей потеря энергии составит

$$
\begin{gathered}
\Delta W_{2}=\Delta W_{\text {раб.1 }}-\Delta W_{\text {раб.з }}= \\
=516500-208000=308500 \mathrm{\kappa ВТ} \cdot ч,
\end{gathered}
$$

или $148 \%$ от оптимального режима.

Соответственно стоимость избыточных потерь электроэнергии будет равна:

$$
\begin{gathered}
C_{1}=139000 \cdot 5,05=701950 \text { р. } / \Gamma ; \\
C_{2}=308500 \cdot 5,05=1557925 \text { p. } / \Gamma,
\end{gathered}
$$

где 5,05 - стоимость 1 кВт.ч для обогатительной фабрики в 2019 г.

Определим потери электроэнергии в трансформаторах ГПП. В нормальном режиме работы комбината один трансформатор является рабочим, другой - резервным. Обмотка низшего напряжения трансформатора 6 кВ питает обогатительную фабрику, а обмотка среднего напряжения 10 кВ - карьер и все вспомогательные подразделения фабрики.
Анализируя данным по расходу электроэнергии и показания счетчиков максимумов на ГПП за период с 2011 по 2019 гг., можно сделать вывод, что наблюдается постоянная тенденция роста электропотребления. Но с 2016 г. процесс стабилизировался, и максимальная мощность за 2019 г. составляет 10,5 МВт, а средняя мощность за этот же год:

$$
P_{\mathrm{cp} 30}=\frac{10,5+8,375+7,863+9,382}{4}=9 \text { МBT. }
$$

На ГПП установлены два трехобмоточных трансформатора ТДТН-25000-110/10/6 с номинальной мощностью $S_{\text {ном }}=25000 \mathrm{MBA}$.

C допущением на дальнейший рост мощности комбината можно утверждать, что трансформатор загружен на половину своей мощности и коэффициент загрузки обмотки высокого напряжения равен $k_{3} \mathrm{BH}=0,5$.

Также на основании данных по учету электроэнергии на ГПП определяем, что потребление электроэнергии на стороне 10 кВ составляет примерно $40 \%$ от общего электропотребления и поэтому коэффициенты загрузок обмоток среднего и низкого напряжения составят соответственно $k_{3 \mathrm{CH}}=0,2 ; k_{3 \mathrm{HH}}=0,3$. Из паспортных данных трансформатора определяем потери холостого хода и короткого замыкания: $\Delta P_{\text {хх }}=36$ кВт; $\Delta P_{\text {кз. вн-нн }}=145$ кВт; $\Delta P_{\text {кз. ВН-сн }}=72,5$ кВт; $\Delta P_{\text {кз. сн-нн }}=72,5$ кВт. На основании годовых данных расхода электроэнергии определяем максимальное число часов работы $T_{\max }$ :

$$
T_{\max }=\frac{W_{\text {год }}}{P_{30 \max }} .
$$

- $\quad$ за 2016 г.: $T_{\max }=\frac{59358600}{10500}=5700$ ч;

- $\quad$ за 2017 г.: $T_{\max }=\frac{66255000}{10500}=6300$ ч;

- за 2018 г.: $T_{\max }=\frac{68317800}{10500}=6500$ ч;

- $\quad$ за 2019 г.: $T_{\max }=\frac{71200800}{10500}=6800$ ч.

Принимаем $T_{\max }=7000$ ч. Тогда $\tau=5700$ ч.

Определим потери мощности в металле обмоток по (17)-(19):

$$
\begin{gathered}
\Delta P_{\text {кз. ВН }}=\frac{72,5+145-72,5}{2}=72,5 \text { кВТ; } \\
\Delta P_{\text {кз. Сн }}=\frac{72,5+72,5-145}{2}=0 \text { кВт; } \\
\Delta P_{\text {кз. НН }}=\frac{145+72,5-72,5}{2}=72,5 \text { кВт. }
\end{gathered}
$$

Потери энергии в трансформаторах определим по формуле (16) для двух случаев: а) работает один трансформатор; б) оба трансформатора работают параллельно.

a)

$$
\begin{gathered}
\Delta W_{1}=2 \cdot 36 \cdot 7000+ \\
+\left[0,5^{2} \cdot 72,5+0,3^{2} \cdot 72,5\right] \cdot 5700= \\
=392505 \text { кВТ } \cdot \text { ч. }
\end{gathered}
$$


б)

$$
\begin{gathered}
\Delta W_{2}=2 \cdot 36 \cdot 7000+ \\
+\left[\left(\frac{0,5}{2}\right)^{2} \cdot 72,5+\left(\frac{0,3}{2}\right)^{2} \cdot 72,5\right] \cdot 5700= \\
=539126 \text { кВт } \div .
\end{gathered}
$$

Стоимость потерь электроэнергии составит соответственно:

$$
\begin{aligned}
& C_{1}=392505 \cdot 5,05=1982150,2 \mathrm{p} . / \Gamma ; \\
& C_{2}=539126 \cdot 5,05=2722586,3 \text { p. } / \Gamma .
\end{aligned}
$$

Тогда при работе одного трансформатора и двух ниток кабеля стоимость потерь будет равна:

$$
C_{1}=(516500+392505) \cdot 5,05=4590475,2 \text { р. } / \Gamma .
$$

При работе одного трансформатора и трех ниток кабеля стоимость потерь будет равна:

$$
C_{2}=(347000+392505) \cdot 5,05=3734500,2 \mathrm{p} . / \Gamma .
$$

При работе двух трансформаторов:

$$
C_{3}=(208000+539126) \cdot 5,05=3772986,3 \mathrm{p} . / \Gamma \text {. }
$$

Как видно, наиболее экономичным является режим работы одного трансформатора и трех ниток кабеля.

Выясним целесообразность замены на ГПП существующих трансформаторов ТДТН-25000 на трансформаторы ТДТН-16000.

В этом случае коэффициенты загрузок обмоток трансформатора будут равны: $k_{3 \mathrm{BH}}=0,7 ; \quad k_{3 \mathrm{CH}}=0,3$ $k_{3 н \mathrm{H}}=0,4$. Из паспортных данных трансформатора определяем потери холостого хода и короткого замыкания: $\Delta P_{\mathrm{xx}}=26$ кВт; $\Delta P_{\mathrm{\kappa} 3}$ ВН-нН $=105$ кВт; $\Delta P_{\text {кзВН-СН }}=52,5 \kappa \mathrm{\kappa T} ;$ $\Delta P_{\text {кз СН-нН }}=52,5$ кВт. Потери мощности в металле обмоток по выражениям (17)-(19): $\Delta P_{\text {кз.вН }}=52,5$ кВт; $\Delta P_{\text {кз.СН }}=0$ кВт; $\Delta P_{\text {кз.нН }}=52,5$ кВт.

Потеря энергии в трансформаторе определится по формуле:

$$
\begin{gathered}
\Delta W=26 \cdot 7000+\left[0,7^{2} \cdot 52,5+0,4^{2} \cdot 52,5\right] \cdot 5700= \\
=376512 \text { кВТ } \cdot \text { ч. }
\end{gathered}
$$

Стоимость потерь электроэнергии составит:

$$
C=376512 \cdot 5,05=1901385,6 \text { р./г. }
$$

Если принять, что при замене ежегодные издержки на обслуживание трансформаторов останутся такими же, то суммарные затраты на демонтаж трансформаторов ТДТН-25000 и установку трансформаторов ТДТН-16000 с учетом срока окупаемости последних будут равны:

$$
\begin{gathered}
3=0,12 \Delta K, \\
\Delta K=K-\left(K_{1}-K_{1} N_{\mathrm{a}} n\right),
\end{gathered}
$$

где $K$ - стоимость новых трансформаторов; $K_{1}$ - стоимость старых трансформаторов; $N_{\mathrm{a}}$ - норма амортизации; $n$ - число лет работы трансформаторов.

$$
\begin{gathered}
3=0,12 \times \\
\times[3900000-(4800000-4800000 \cdot 0,064 \cdot 6)]= \\
=113184 \text { p./г. }
\end{gathered}
$$

Таким образом, затраты на монтажные работы превышают величину экономии электроэнергии, получаемую в результате замены трансформаторов:
$\ni_{\Delta W}=C_{2}-C=3734500,2-1901385,6=1833114,6$ p.

В данном случае замена существующих трансформаторов на трансформаторы меньшей мощности нецелесообразна.

\section{Выводы}

1. Разработана методика определения потерь электроэнергии в элементах системы электроснабжения в номинальном и в эксплуатационном режиме.

2. Отношение коэффициентов потерь в номинальном и эксплуатационном режимах работы, определяющее степень использования пропускной способности кабельной сети напряжением 6 киловольт, находится в пределах 1,02-2,57. В среднем кабельная сеть недогружена на 25-30\%.

3. Фактические потери электроэнергии в кабельной сети напряжением 6 кВ оказались меньше номинальных и составляют менее $1 \%$ от общей потребляемой электроэнергии, следовательно, проведение каких-либо мероприятий для уменьшения потерь электроэнергии нецелесообразно.

4. Средневзвешенный коэффициент мощности по обогатительной фабрике равен 0,82 , поэтому может оказаться целесообразным проведение мероприятий по компенсации реактивной мощности. Расчет компенсирующих устройств и выбор места их установки предусмотрены планом работ на будущий период.

5. Для снижения потерь электроэнергии в кабельных линиях от трансформаторов ГПП до ГРУ целесообразно, чтобы в работе находились оба ввода и кабельные линии работали параллельно. При работе одного ввода стоимость дополнительных потерь электроэнергии составляет 1557925 р./г.

6. Коэффициенты загрузки трансформаторов ГПП равны: $k_{3 \mathrm{BH}}=0,5 ; k_{3 \mathrm{CH}}=0,2 ; k_{3 \mathrm{HH}}=0,3$. Однако замена их на трансформаторы меньшей мощности нецелесообразна в связи с высокой стоимостью монтажных работ. При плановой замене трансформаторов ГПП и существующем уровне энергопотребления рекомендуется замена трансформаторов ТДТН-25000 на ТДТН-16000. При этом будет получен экономический эффект:

$$
\begin{gathered}
Э=Э_{\Delta W}+Э_{\Delta K}= \\
=1833114,6+0,12 \cdot(4800000-3900000)= \\
=1941114,6 \text { р. } / \text { г. }
\end{gathered}
$$

7. Проведен анализ потерь электроэнергии в электрической сети обогатительной фабрики в условиях горных территорий, характеризующихся сложными климатическими условиями и необходимостью обеспечения эффективного энергообеспечения труднодоступных отдаленных потребителей электроэнергии. Новизна разработанной методики заключается в получении новых данных по потерям мощности для отдельных потребителей фабрики (дробилки, шаровые мельницы, насосы и т. д.), а также в установлении необходимости проведения комплексных мероприятий по компенсации реактивной мощности. 


\section{СПИСОК ЛИТЕРАТУРЫ}

1. Голик В.И., Разоренов Ю.И., Ляшенко В.И. Условия выщелачивания цветных металлов из забалансового сырья // Известия Томского политехнического университета. Инжиниринг георесурсов. - 2018. - Т. 329. - № 6. - С. 6-16.

2. Biel K., Glock C. Systematic literature review of decision support models for energy-efficient production planning // Computers \& Industrial Engineering. - 2016. - V. 101. - P. 243-259.

3. Bornschlegl M., Bregulla M., Franke J. Methods-Energy Measurement - an approach for sustainable energy planning of manufacturing technologies // Journal of Cleaner Production. 2016. - V. 1351. - P. 644-656.

4. Motivation towards energy saving by means of IoT personal energy manager platform / Y. Zhukovskiy, D. Batueva, A. Buldysko, M. Shabalov // Journal of Physics: Conference Series. 2019. - 1333 (6). DOI: 10.1088/1742-6596/1333/6/062033.

5. Комплексный анализ применения эффективных технологий для повышения устойчивого развития природно-технической системы / Р.В. Клюев, И.И. Босиков, А. В. Майер, О.А. Гаврина // Устойчивое развитие горных территорий. - 2020. - № 2. C. $283-290$.

6. Improving the energy efficiency of technological equipment at mining enterprises / R. Klyuev, I. Bosikov, O. Gavrina, M. Madaeva, A. Sokolov // Advances in Intelligent Systems and Computing. - 2021. -V. 1258. - P. 262-271.

7. Energy indicators of drilling machines and excavators in mountain territories / R. Klyuev, O. Fomenko, O. Gavrina, R. Turluev, S. Marzoev // Advances in Intelligent Systems and Computing. 2021. -V. 1258. - P. 272-281.

8. Стефанов В.К., Антоненков Д.В., Игумнова С.А. Удельный расход электроэнергии карьерных экскаваторов работающих в условиях крайнего Севера // Горный информационноаналитический бюллетень. - 2011. - № S4. - С. 32-38.

9. Ляхомский А.В., Петухов С.В. Энергоэффективность экскаваторных работ угледобывающих предприятий // Промышленная энергетика. - 2019. - № 1. - С. 38-41.
10. Wang J., Du Yu, Wang J. LSTM based long-term energy consumption prediction with periodicity // Energy. - 2020. V. 197. - Article 117197.

11. Wang R., Lu Sh., Feng W. A novel improved model for building energy consumption prediction based on model integration // Applied Energy. - 2020. - V. 262. - Article 114561.

12. Farhadi P. Golmohammadi, A., Malvajerdi A., Shahgholi G. Tire and soil effects on power loss: measurement and comparison with finite element model results // Journal of Terramechanics. 2020. - V. 92. - P. 13-22.

13. Distribution networks nontechnical power loss estimation: A hybrid data-driven physics model-based framework / A. Bretas, A. Rossoni, R. Trevizan, N. Bretas // Electric Power Systems Research. - 2020. - V. 186. - Article 106397.

14. Nappu M., Arief A. Network losses-based economic redispatch for optimal energy pricing in a congested power system // Energy Procedia. - 2016. - V. 100. - P. 311-314.

15. Sizing energy storage systems in DC networks: a general methodology based upon power losses minimization / M. Fantauzzi, D. Lauria, F. Mottola, A. Scalfati // Applied Energy. 2017. - V. 187. - P. 862-872.

16. Hartmann B., Farkas C. Energy efficient data centre infrastructure - development of a power loss model // Energy and Buildings. 2016. - V. 127. - P. 692-699.

17. Routray A., Mistry K., Arya S. Wake analysis on wind Farm power generation for loss minimization in radial distribution system // Renewable Energy Focus. - 2020. - V. 34. - P. 99-108.

18. Davidov S., Pantoš M. Optimization model for charging infrastructure planning with electric power system reliability check // Energy. - 2019. - V. 166. - P. 886-894.

19. Afzali P., Keynia F., Rashidinejad M. A new model for reliabilitycentered maintenance prioritisation of distribution feeders // Energy. - 2019. - V. 171. - P. 701-709.

20. Rebello S., Yu H., Ma L. An integrated approach for system functional reliability assessment using dynamic bayesian network and hidden Markov model // Reliability Engineering \& System Safety. - 2018. - V. 180. - P. 124-135

Поступила 16.06.2021 2.

\section{Информация об авторах}

Клюев Р.В., доктор технических наук, профессор кафедры техники низких температур им. П.Л. Капицы Московского политехнического университета.

Голик В.И., доктор технических наук, профессор кафедры технологии разработки месторождений СевероКавказского горно-металлургического института (государственного технологического университета).

Босиков И.И., кандидат технических наук, доцент кафедры прикладной геологии Северо-Кавказского горнометаллургического института (государственного технологического университета).

Гаврина О.А., кандидат технических наук, доцент кафедры электроснабжения промышленных предприятий Северо-Кавказского горно-металлургического института (государственного технологического университета). 
UDC 621.311

\title{
ANALYSIS OF ELECTRIC POWER LOSS IN THE POWER SUPPLY SYSTEM OF THE CONCENTRATING FACTORY
}

\author{
Roman V. Klyuev 1 , \\ kluev-roman@ramler.ru \\ Vladimir I. Golik², \\ v.i.golik@mail.ru \\ Igor I. Bosikov2, \\ igor.boss.777@mal.ru \\ Oksana A. Gavrina², \\ Gavrina-Oksana@yandex.ru \\ ${ }^{1}$ Moscow Polytechnic University, \\ 33, B. Semenovskaya street, Moscow, 107023, Russia. \\ 2 North Caucasian Institute of mining and metallurgy (State Technological University), \\ 44, Nikolaev street, Vladikavkaz, 362021, Russia.
}

The relevance of the work is caused by the need for a comprehensive study of the power supply system of the processing plants. For this, it is necessary to develop an effective methodology for calculating electricity losses for individual technological mechanisms and the factory as a whole, which will make it possible to significantly reduce the energy component of costs during the production process.

The main aim of the research is to develop a methodology for determining energy losses by individual technological mechanisms in various operating modes, which can be used at similar enterprises.

Objects: mining and concentrating factories, which are characterized by the complexity of determining the energy characteristics of technological equipment.

Methods: a combination of deterministic and probabilistic-statistical methods for determining energy losses. Deterministic methods include calculating maximum losses, average loads, graphical integration, and rms current. The probabilistic statistic ones, in their turn, are based on the study of statistical characteristics and the use of statistical samples of network schemes

Results. Based on the experimental studies, a methodology has been developed for determining the energy losses in the elements of the power supply system of the concentrating factory in nominal and operational mode. It was established that the ratio of loss factors in the nominal and operational modes of operation, which determines the degree of utilization of the capacity of the cable network of $6 \mathrm{kV}$, is in the range of 1,02-2,57. On average, a $6 \mathrm{kV}$ cable network is underloaded by 25-30\%. Actual electricity losses in the $6 \mathrm{kV}$ cable network turned out to be less than the nominal ones and make up less than $1 \%$ of the total electricity consumed, therefore, any measures to reduce electricity losses are impractical. The weighted average power factor for the concentration plant is 0,82 , so it may be appropriate to carry out measures to compensate the reactive power. The calculation of the parameters of the compensating devices and the choice of their installation site are provided for by the work plan for the future period.

\section{Key words:}

Processing plant, electricity losses, cable network, power supply system, graphic integration method, equipment capacity.

\section{REFERENCES}

1. Golik V.I., Razorenov Yu.I., Lyashenko V.I. Conditions for leaching non-ferrous metals from off-balance materials. Bulletin of the Tomsk Polytechnic University. Geo Assets Engineering, 2018, vol. 329, no. 6, pp. 6-16. In Rus.

2. Biel K., Glock C. Systematic literature review of decision support models for energy-efficient production planning. Computers \& Industrial Engineering, 2016, vol. 101, pp. 243-259.

3. Bornschlegl M., Bregulla M., Franke J. Methods-Energy Measurement - an approach for sustainable energy planning of manufacturing technologies. Journal of Cleaner Production, 2016, vol. 1351, pp. 644-656.

4. Zhukovskiy Y., Batueva D., Buldysko A., Shabalov M. Motivation towards energy saving by means of IoT personal energy manager platform. Journal of Physics: Conference Series, 2019, 1333 (6). DOI: 10.1088/1742-6596/1333/6/062033.

5. Klyuev R.V., Bosikov I.I., Mayer A.V., Gavrina O.A. Comprehensive analysis of the effective technologies application to increase sustainable development of the natural-technical system. Sustainable Development of Mountain Territories, 2020, no.2, pp. 283-290. In Rus.

6. Klyuev R., Bosikov I., Gavrina O., Madaeva M., Sokolov A. Improving the energy efficiency of technological equipment at mining enterprises. Advances in Intelligent Systems and Computing, 2021, vol. 1258, pp. 262-271.

7. Klyuev R., Fomenko O., Gavrina O., Turluev R., Marzoev S. Energy indicators of drilling machines and excavators in mountain territories. Advances in Intelligent Systems and Computing, 2021, vol. 1258, pp. 272-281.

8. Stefanov V.K., Antonenkov D.V., Igumnova S.A. Specific power consumption of quarry excavators operating in the far North. MIAB. Mining Inf. Anal. Bull., 2019, no (S4), pp. 32-38. In Rus.

9. Lyakhomsky A.V., Petuhov S.V. Energy efficiency of excavating operations of coal mining enterprises. Promyshlennaya energetika, 2019, no. 1, pp. 38-41. In Rus.

10. Wang J., Du Yu, Wang J. LSTM based long-term energy consumption prediction with periodicity. Energy, 2020, vol. 197, Article 117197.

11. Wang R., Lu Sh., Feng W. A novel improved model for building energy consumption prediction based on model integration. Applied Energy, 2020, vol. 262, Article 114561.

12. Farhadi P., Golmohammadi A., Malvajerdi A., Shahgholi G. Tire and soil effects on power loss: Measurement and comparison with finite element model results. Journal of Terramechanics, 2020, vol. 92. pp. 13-22.

13. Bretas A., Rossoni A., Trevizan R., Bretas N. Distribution networks nontechnical power loss estimation: A hybrid data- 
driven physics model-based framework. Electric Power Systems Research, 2020, vol. 186, Article 106397.

14. Nappu M., Arief A. Network Losses-Based Economic Redispatch For Optimal Energy Pricing In A Congested Power System. Energy Procedia, 2016, vol. 100, pp. 311-314.

15. Fantauzzi M., Lauria D., Mottola F., Scalfati A. Sizing energy storage systems in DC networks: A general methodology based upon power losses minimization. Applied Energy, 2017, vol. 187, pp. $862-872$.

16. Hartmann B., Farkas C. Energy efficient data centre infrastructure-Development of a power loss model. Energy and Buildings, 2016, vol. 127, pp. 692-699.

17. Routray A., Mistry K., Arya S. Wake analysis on wind Farm power generation for loss minimization in radial distribution system. Renewable Energy Focus, 2020, vol. 34, pp. 99-108.
18. Davidov S., Pantoš M. Optimization model for charging infrastructure planning with electric power system reliability check. Energy, 2019, vol. 166, pp. 886-894.

19. Afzali P., Keynia F., Rashidinejad M. A new model for reliabilitycentered maintenance prioritisation of distribution feeders. Energy, 2019, vol. 171, pp. 701-709.

20. Rebello S., Yu H., Ma L. An integrated approach for system functional reliability assessment using dynamic bayesian network and hidden Markov model. Reliability Engineering \& System Safety, 2018, vol. 180, pp. 124-135.

Received: 16 June 2021.

\section{Information about the authors}

Roman V. Klyuev, Dr. Sc., chief of industrial power supply department, Moscow Polytechnic University.

Vladimir I. Golik, Dr. Sc., professor, North Caucasian Institute of mining and metallurgy (State Technological University).

Igor I. Bosikov, Cand. Sc., assistant professor, North Caucasian Institute of mining and metallurgy (State Technological University).

Oksana A. Gavrina, Cand. Sc., assistant professor, North Caucasian Institute of mining and metallurgy (State Technological University). 